Bangladesh J. Sci. Res. 25(1): 73-86, 2012 (June)

\title{
NON-LINEAR AGE-TIME-POPULATION DEPENDENT STOCHASTIC POPULATION MODEL
}

\author{
Md. Jamal Hossain and Md. Shahidul Islam* \\ Department of Mathematics, University of Dhaka, Dhaka-1000, Bangladesh
}

\begin{abstract}
In this paper, we study a non-deterministic Gurtin-MacCamy Population model, a model in which a random environment may affect the system. Since the environment changes are due to many factors, we can approximate by noise (that is, a Gaussian white noise), although we do not know the exact behavior of the noise term. By allowing some randomness in the system, we often obtain a more realistic mathematical model of the population. We investigate global stability in presence of noise, the long term behavior of the dynamical system.
\end{abstract}

Key words: Non-linear, population model, age-time population, Brownian motion, stochastic model.

\section{Introduction}

During the last 40 years, interest in the study of stochastic phenomena has increased dramatically. Intensified research activity in this area has been stimulated by the need to take into account random effects in complicated physical systems. The first random model was formulated by Langevin in 1908 to describe the velocity of a particle moving in a random force field. Random equations of this type can be interpreted as stochastic differential equations, following Itô's basic work in the early 1940s. Solutions of such equations represent Markov diffusion processes, which is the Brownian motion.

The first mathematical model was given by Malthusian (Murray 2002) known as Malthusian model. Then Verhulst (Murray 2002), also known as Logistic model, introduced carrying capacity and modify Malthusian model. The growth of an agestructured single species population model was first proposed by Sharpe and Lotka (Sharpe and Lotka 1911). This model known as the Lotka-Von Foerster model is discussed in the literature (Foerster 1959) and is based on age-specific natality and mortality rates which are independent of time and population. Langhaar (1972) considered the dependence of individual mortality and reproduction on time explicitly and Gurtin and MacCamy (1974) discussed the dependence of birth and death moduli on the population. We consider a model in the age-time-population in which the dependence of natality and mortality rates on age, time and population are incorporated and allowing some randomness in the system. Thus the model under consideration is an extension of the non-linear model of Gurtin and MacCamy (1974).

\footnotetext{
Corresponding author, E-mail: mshahidull1@yahoo.com
} 
We study a non-linear Gurtin-MacCamy model into stochastic population model and construct feasible solutions in the approach of stochastic integral and Brownian motion and discuss the behavior on the growth rate and death rate of population.

\section{Preliminaries}

Let $(\Omega, \boldsymbol{A}, P)$ be a probability space, consisting of sample space $\Omega$, a $\sigma$-algebra $\boldsymbol{A}$ of subsets of $\Omega$ called events, and a real-valued function $P$ defined on $A$ is called a probability.

A stochastic process or random process is the counterpart to a deterministic process in probability theorem. Instead of dealing with possible 'reality', the process might involve under time in a stochastic or random process there is some indeterminacy in its future evolution described by probability distributions. Familiar examples of process model of stochastic time series include stock market and exchange rate fluctuations, signals such as speech, audio and video, medical data such as a patient's EEG, blood pressure or temperature, and random movement such as Brownian motion or random walks.

Definition 1. (i) A random variables $\{X(t) \mid t \geq 0\}$ is called a stochastic process.

(ii) For each point $\omega \in \Omega$ the mapping $t \rightarrow X(t, \omega)$ is the corresponding sample path.

If we run an experiment and observe the random values of $X(\cdot)$ as time evolves, we are in fact looking at a sample path $\{X(t, \omega) \mid t \geq 0\}$ for some fixed $\omega \in \Omega$.

A stochastic process $\{X(t): a \leq t \leq b\}$ on a probability space $(\Omega, A, P)$ is called a martingale with respect to non-decreasing family $\{A(t): a \leq t \leq b\}$ of sub $\sigma$-algebra of $A$, if for each $t \in[a, b], X(t)$ is $A(t)$-measurable, $E(|X(t)|)<\infty$ and for each $s>0$.

Then

$$
E(X(t+s) \mid A(t))=X(t) .
$$

The irregular motions of small particles of colloidal size immersed in a fluid were first noticed by the British botanist Brown in 1826. The chaotic motion of such a particle is called Brownian motion (Gard 1988) and a particle is called a Brownian particle.

Definition 2. A real-valued stochastic process $W(\cdot)$ is called a Brownian motion or Wiener process if

(i) $W(0)=0$ almost surely, (ii) $W(t)-W(s)$ is $N(0, t-s)$ for all $t \geq s \geq 0$, 
(iii) for all times $0<t_{1}<t_{2}<\cdots<t_{n}$, the random variables $W\left(t_{1}\right), W\left(t_{2}\right)-W\left(t_{1}\right)$, $\cdots, W\left(t_{n}\right)-W\left(t_{n-1}\right)$ are independent (independent increments).

In particular that

$$
E(W(t))=0, E\left(W^{2}(t)\right)=t \text { for each time } t \geq 0 .
$$

\section{Standard Brownian Motion}

A standard Brownian (or a standard Wiener process) is a stochastic process $\left\{W_{t}\right\}_{t \geq 0}$, that is, a family of random variables $W_{t}$, indexed by nonnegative real numbers $t$, defined on a common probability space $(\Omega, F, P)$ with the following properties:

(i) $W_{0}=0$

(ii) With probability 1 , the function $t \rightarrow W_{t}$ is continuous in $t$.

(iii) The process $\left\{W_{t}\right\}_{t \geq 0}$ has the normal $(0, t)$ distribution.

The term independent increments means that for every choice of non-negative real numbers $0 \leq s_{1} \leq t_{1} \leq s_{2} \leq t_{2} \leq \ldots \leq s_{n} \leq t_{n} \leq \infty$, the increment of random variable $W_{t_{1}}-W_{s_{1}}, W_{t_{2}}-W_{s_{2}}, \ldots \ldots ., W_{t_{n}}-W_{s_{n}}$ are jointly independent, the term stationary increments means that for any $0<s, t<1$ the distribution of the increment $W_{t+s}-W_{s}$ has the same distribution as $W_{t}-W_{0}=W_{t}$.

\section{Stochastic Differential Equations}

Let $W(\cdot)$ be an $m$-dimensional Brownian motion and $X_{0}$ an $n$-dimensional random variable which is independent of $W(\cdot)$ and $F(t):=U\left(X_{0}, W(s)\right), 0 \leq s \leq t$ the $\sigma$ algebra generated by $X_{0}$ and the history of the Wiener process up to time $t$.

Definition 3. We say that an $\Re^{n}$-valued stochastic process $X(\cdot)$ is a solution of the Itô stochastic differential equation

$$
\left\{\begin{array}{l}
d X=b(X, t) d t+B(X, t) d W \\
X(0)=X_{0}
\end{array}\right.
$$

for $0 \leq t \leq T$, provided 
(i) $X(\cdot)$ is progressively measurable with respect to $F(\cdot)$,

(ii) $F:=b(X, t) \in L_{n}^{1}(0, T)$,

(iii) $G:=B(X, t) \in L_{n \times m}^{2}(0, T)$ and

(iv) $X(t)=X_{0}+\int_{0}^{t} b(X(s), s) d s+\int_{0}^{t} B(X(s), s) d W$ almost surely for all $0 \leq t \leq T$.

Itô's Integral Formula

Let $W(\cdot)$ be a 1-dimensional Brownian motion defined on probability space $(\Omega, U, P)$.

Suppose that $X(\cdot)$ has a stochastic differential $d X=F d t+G d W$, for $F \in L^{1}(0, T)$

and $G \in L^{2}(0, T)$.

Assume $u: R \times[0, T] \rightarrow \Re$ is continuous and $\frac{\partial u}{\partial t}, \frac{\partial u}{\partial x}, \frac{\partial^{2} u}{\partial x^{2}}$ exist and are continuous.

Set $Y(t):=u(X(t), t)$. Then $Y$ has the stochastic differential (Grad 1988)

$$
d Y=\left(\frac{\partial u}{\partial t}+\frac{\partial u}{\partial x} F+\frac{1}{2} \frac{\partial^{2} u}{\partial x^{2}} G^{2}\right) d t+\frac{\partial u}{\partial x} G d W .
$$

\section{Gurtin-MacCamy Model}

In 1974, Gurtin and MacCamy introduced a more realistic formulation of a nonlinear, continuous, age-dependent, deterministic population model. They studied the effect of crowding appears in the model, for the fertility and mortality moduli are allowed to be nonlinear functional of the population at any time. The problem is based on the following initial-boundary value problem:

$$
\left\{\begin{array}{l}
\frac{\partial P(a, t)}{\partial a}+\frac{\partial P(a, t)}{\partial t}+\mu(a, P(t)) P(a, t)=0, a>0, t>0 \\
P(0, t)=\int_{0}^{\infty} \beta(a, P(t)) P(a, t) d a, t \geq 0 \\
P(a, 0)=\varphi(a), a \geq 0 \\
P(t)=\int_{0}^{\infty} P(a, t) d a, t \geq 0
\end{array}\right.
$$


where $P(a, t)$ is the density of population with respect to the age $a \in[0, \infty)$ at time $t \geq 0$ and $P(t)$ is the total population size at time $t ; \beta(a, P(t)), \mu(a, P(t))$ are respectively, the birth rate and the mortality rate when the population size is $P(t)$.

\section{Stochastic Differential Equations of Gurtin-MacCamy Model}

In recent years, it has become apparent that physical systems can be more satisfactorily modeled by certain stochastic counterparts if random effects in the physical phenomena as well as measuring devices are to be taken into account. Gurtin-MacCamy model can be written in the form of stochastic differential equation (SDE) as follows:

$$
\left\{\begin{array}{l}
\frac{\partial P(a, t)}{\partial a}+\frac{\partial P(a, t)}{\partial t}+\mu(a, P(t)) W(t)=0, a>0, t>0 \\
P(0, t)=\int_{0}^{t} \beta(a, P(t)) d W(a), t \geq 0 \\
P(a, 0)=P_{0}(a), a \geq 0 \\
W(t)=\int_{0}^{t} P(a, t) d W(a), t \geq 0
\end{array}\right.
$$

where $W(t)$ is Wiener process.

\section{Linear Case}

We rewrite the above model as follows:

$$
\left\{\begin{array}{l}
P_{a}(a, t)+P_{t}(a, t)=-\mu(a, P(t)) W(t), t>0, a>0 \\
P(0, t)=K F(t), t>0 \\
F(t)=A-\int_{0}^{t} \beta(a, P(t)) d W(a), t>0 \\
P(a, 0)=P_{0}(a), a \geq 0 \\
W(t)=\int_{0}^{t} P(a, t) d W(a), t \geq 0 .
\end{array}\right.
$$

It is assume that $\beta \in L_{+}^{\infty}(0, t), \mu(a, P(t))$ is positive for all $a \in[0, t]$, locally integrable on $[0, t)$ and it follows that

$$
\int_{0}^{t} \mu(a, P(t)) d W(a)=\infty .
$$


The condition (5) is needed to make the maximum attainable age to be finite. The survival rate (the proportion of newly born who can survive to age $a$ ) is given by .

$$
l(a):=\exp \left\{-\int_{0}^{t} \mu(a, P(t)) d W(a)\right\}
$$

Under the above assumption, the natural state space $\Omega$ of the age density function may be

$$
\Omega:=\left\{P \in L_{+}^{1}(0, t): \int_{0}^{i} \mu(a, P(t)) d W(a)<\infty, \int_{0}^{t} \beta(a, P(t)) d W(a) \leq A\right\} .
$$

First, in order to avoid mathematical troubles due to the singularity of the mortality rate $\mu$, let us factor out the natural death rate in the basic model (4). Define a new function $q(a, t)$ by $P(a, t)=l(a) q(a, t)$.

It is easy to see that the system (4) can be reduced to a simple system for $q$ as follows:

$$
\begin{aligned}
& \frac{\partial P(a, t)}{\partial a}+\frac{\partial P(a, t)}{\partial t}=0, a>0, t>0 \\
& q(0, t)=K\left(A-\int_{0}^{t} \varphi(a) d W(a)\right), t>0 \\
& q(a, 0)=\frac{P_{0}(a)}{l(a)} \in L_{+}^{1}(0, t)
\end{aligned}
$$

where $\varphi(a):=\beta(a, P(t)) l(a)$ is the expected space size occupied by the population at age $a$ and we assume that $P_{0} / l \in L_{+}^{1}(0, t)$. We assume that the age density function $t \rightarrow q(*, t)$ takes a value in $L_{+}^{1}(0, t)$. Assume that $\mu l \in L^{\infty}([0, t])$, we have $P=q l \in \Omega$ when $\int_{0}^{t} q(a) \varphi(a) d W(a) \leq A$.

It is easy to see that the model (6), there always exist a unique positive steady state as

$$
q^{*}(a)=\frac{K A}{1+K \int_{0}^{t} \varphi(a) d W(a)} .
$$

In order to rewrite the basic model as a homogeneous boundary condition, let us introduce a variable $u(a, t)$ as $u(a, t)=q(a, t)-q^{*}(a)$. 
Then the system (6) can be rewritten into the following homogeneous system:

$$
\begin{aligned}
& \frac{\partial u(a, t)}{\partial a}+\frac{\partial u(a, t)}{\partial t}=0, a>0, t>0 \\
& u(0, t)=-K \int_{0}^{t} \varphi(a) d W(a), t>0 \\
& u(a, 0)=q_{0}(a)-q^{*}(a) .
\end{aligned}
$$

Therefore, we know that the model is reduced to a linear homogeneous age dependent population system in $L^{1}$ and the well known argument for Lotka's stable population model can be applied to this new system. Integrating (7) along the characteristic line, we obtain the following expression:

$$
u(a, t)=\left\{\begin{array}{l}
b(t-a), t-a>0 \\
u_{0}(a-t), a-t>0
\end{array}\right.
$$

where $b(t):=u(0, t)$. Inserting (8) into (7), we have a renewal integral equation as

$$
b(t)=-g(t)-K \int_{0}^{t} \varphi(a) b(t-a) d W(a),
$$

where $g(t)$ is defined by

$$
g(t):=K \int_{t}^{\infty} \varphi(a) u_{0}(a-t) d W(a) .
$$

We extend the domain of $\varphi(a)$ as $\varphi(a)=0$ for $a>t$. Let $\Lambda$ be the set of characteristic roots as $\Lambda:=\{\lambda \in C: 1+K \hat{\varphi}(\lambda)=0\}$

where $\hat{\varphi}$ denotes the Laplace transform of $\varphi$ given by

$$
\hat{\varphi}(\lambda):=\int_{0}^{\infty} e^{-\lambda a} \varphi(a) d W(a) .
$$

Proposition 1. Let $\Lambda \cap \Re=\varphi$ and $\Lambda$ is composed of countable infinite number of complex conjugate pairs. For any real number $\alpha$, there is at most finitely many roots in the right half plane $\Re \lambda>\alpha$, then there is a dominant pair whose real part is greater than real part of any other characteristic root. 
On the other hand the solution of (9) can be obtained by the inverse Laplace transformation:

$$
b(t)=-\frac{1}{2 \pi i} \int_{\sigma-i \infty}^{\sigma+i \infty} \frac{\hat{g}(\lambda) e^{\lambda t}}{1+K \hat{\varphi}(\lambda)} d W(\lambda)
$$

where $\sigma$ is real number such that $\sigma>\max \{\Re \lambda: \lambda \in \Lambda\}$. Let $\lambda_{0}$ and $\bar{\lambda}_{0}$ be the dominant pair. It follows that

$$
\begin{aligned}
b(t) & =b_{0} e^{\lambda_{0} t}+\bar{b}_{0} e^{\bar{\lambda}_{0 t}}+O\left(e^{\left(\Re \lambda_{0}-\varepsilon\right) t}\right) \\
& =e^{\Re \lambda_{0} t}\left[\Re b_{0} \cos \left(\operatorname{Im} \lambda_{0} t\right)-\operatorname{Im} b_{0} \sin \left(\operatorname{Im} \lambda_{0} t\right)\right]+O\left(e^{\left(\Re \lambda_{0}-\varepsilon\right) t}\right)
\end{aligned}
$$

where $\varepsilon>0$ is a small number such that

$$
\left\{\lambda: \lambda \in \Lambda \backslash\left\{\lambda_{0}, \bar{\lambda}_{0}\right\}\right\} \subset\left\{\lambda: \Re \lambda \leq \Re \lambda_{0}-\varepsilon\right\},
$$

and $b_{0}$ is given by

$$
b_{0}:=\frac{\int_{0}^{t} e^{-\lambda_{0} t} g(s) d W(s)}{K \int_{0}^{t} a e^{-\lambda_{0} a} \varphi(a) d W(a)} .
$$

Hence asymptotically the dominant part of the solution of the basic model (4) is given

$$
l(a) q^{*}(a)+\left|b_{0}\right| e^{\Re \lambda_{0}(t-a)} l(a) \cos \left(\operatorname{Im} \lambda_{0}(t-a)+\theta\right), \text { where } \theta:=\arctan \left(\operatorname{Im} b_{0} / \Re b_{0}\right) .
$$

Then there is no Malthusian solution, and the steady state is globally asymptotically stable if $\Re \lambda_{0}<0$, whereas it is unstable if $\Re \lambda_{0}>0$. In particular, if $\Re \lambda_{0}>0$, the positivity of the population density will be destroyed as time passes.

\section{Nonlinear Case}

We extend the basic linear model into a nonlinear model such that it has the density dependent mortality. Let $\mu(a, P(t))$ be the natural death rate at age $a$ and $\delta(a, S(t))$ be the extra death rate of population with free space $A-S$ and age $a$, where $S(t)$ denote the size of occupied space 


$$
S(t):=\int_{0}^{t} \beta(a, P(t)) d W(a)
$$

Then the non-linear model is formulated as follows:

$$
\left\{\begin{array}{l}
\frac{\partial P(a, t)}{\partial a}+\frac{\partial P(a, t)}{\partial t}=-(\mu(a, S(t))+\delta(a, S(t))) W(t), a>0, t>0 \\
P(0, t)=K(A-S(t)), t \geq 0 \\
P(a, 0)=P_{0}(a), a \in[0, t] \\
W(t)=\int_{0}^{t} P(a, t) d W(a), t \geq 0
\end{array}\right.
$$

where $P_{0}$ is a given initial data.

If we define formally the size-dependent age-specific birth rate $m(a, S)$ by

$$
m(a, S):=\frac{K(A-S)}{S} \beta(a, S(t))
$$

then, by Roughgarden et al. (1985), the system (10) can be formally rewritten as the Gurtin and MacCamy's nonlinear age-dependent population model:

$$
\left\{\begin{array}{l}
\frac{\partial P(a, t)}{\partial a}+\frac{\partial P(a, t)}{\partial t}=-(\mu(a, S(t))+\delta(a, S(t))) W(t) \\
P(0, t)=\int_{0}^{t} m(a, S(t)) d W(a) \\
S(t)=\int_{0}^{t} \beta(a, S(t)) d W(a) \\
W(t)=\int_{0}^{t} P(a, t) d W(a) .
\end{array}\right.
$$

Then the demographic basic reproduction number corresponding to the weighted population size $S$, denoted by $R_{0}(S)$, is given as follows:

$$
R_{0}(S)=\frac{K(A-S)}{S} \int_{0}^{t} \varphi(a) \Gamma(a, S(t)) d W(a)
$$

where $\Gamma(a, S):=\exp \left\{-\int_{0}^{a} \delta(a, S) d W(a)\right\}$ 
Due to the singularity of the size-dependent birth rate $m$ at $S=0$, the population with size zero has an infinitely large reproductivity. Hence we cannot apply the general theory of nonlinear age-dependent population dynamics model. We adopt the following reasonable but technical assumptions:

Assumption. (1) The extra death rate $\delta(a, x)$ is positive and uniformly bounded for $(a, x) \in[0, \omega] \times[0, A]$. The domain of $\delta(a, x)$ is formally extended as

$$
\delta(a, x)=\left\{\begin{array}{l}
\delta(a, A), \text { for } x \geq A \\
0, \text { for } x \leq 0
\end{array}\right.
$$

and assume that $\delta(a, x)$ is continuously differentiable with respect to all $x \in \Re$ and there exist numbers $\bar{\delta}$ and $M>0$ such that

$$
C \leq \delta(a, x) \leq \bar{\delta}, 0 \leq \frac{\partial \delta(a, x)}{\partial x} \leq M, \text { for almost all }(a, x) \in[0, t] \times \Re
$$

(2) $\beta$ is differentiable, positive and bounded function on $[0, t]$ and

$$
\mu(a) \beta(a) \leq \beta^{\prime} \leq(\mu(a)+\delta(a, A)) \beta(a),
$$

for almost all $a \in[0, t]$ and $\beta(0, S)=0$.

Just the same as the linear case, in order to make the mathematical treatment easier, let us factor out the natural death rate in the basic model (10). Define a new function $q(a, t)$ by $P(a, t)=l(a) q(a, t)$, where $l(a)$ is the survival function. Then it is easy to see that the system (10) can be reduced to a simpler one as follows:

$$
\left\{\begin{array}{l}
\frac{\partial q(a, t)}{\partial a}+\frac{\partial q(a, t)}{\partial t}=-\delta(a, S(t)) W(t), t>0, a \in[0, t] \\
q(0, t)=K(A-S(t)), t>0 \\
S(t)=\int_{0}^{t} \varphi(a) d W(a), t>0 \\
q(a, 0)=\frac{P_{0}(a)}{l(a)} \in L_{+}^{1}(0, t), t>0 \\
W(t)=\int_{0}^{t} q(a, t) d W(a), t>0
\end{array}\right.
$$


where $\varphi(a):=\beta(a) l(a)$ is the expected space size occupied by the population at age $a$. Naturally, we can define the state space of the new system (13) as

$$
\Omega:=\left\{q \in L_{+}^{1}(0, t): \int_{0}^{t} \varphi(a) q(a) d W(a) \leq A\right\} .
$$

In order to be a biologically meaningful model, the solution of the system (13) must be in the state space $\Omega$ if $q_{0} \in \Omega$. Under the Assumption, if $q_{0} \in \Omega$, the nonnegative solution of the system (13), as long as it exists, will stay in the state space $\Omega$. In fact if we integrate by parts, we obtain that

$$
\frac{d S(t)}{d t}=\int_{0}^{t}\left(\beta^{\prime}(a, S(t))-(\mu(a)+\delta(a, S(t))) \beta(a, S(t))\right) l(a) d W(a) .
$$

Then it follows from Assumption, $S(t)$ is bounded in the interval $[0, A]$ for all $t \geq 0$, because $S^{\prime} \leq 0$ at the neighborhood of $S=A$ and $S^{\prime} \geq 0$ at the neighborhood of $S=0$.

\section{The Semigroup Solution}

First observe that the system (13) has a unique positive steady state. Let $q^{*}(a)$ be a steady state solution for the system (13) and let $S^{*}$ be the corresponding size of occupied space. Then we have

$$
\begin{aligned}
& q^{*}(a)=q^{*}(0) \Gamma\left(a, S^{*}\right) \\
& q^{*}(0)=K\left(A-S^{*}\right)=K\left(A-\int_{0}^{t} \varphi(a) q^{*}(a) d W(a)\right) .
\end{aligned}
$$

It is easy to see that

$$
\begin{aligned}
& R_{0}\left(S^{*}\right)=\frac{K\left(A-S^{*}\right)}{S^{*}} \int_{0}^{t} \varphi(a) \Gamma\left(a, S^{*}\right) d W(a)=1 \\
& q^{*}(a)=\frac{S^{*} \Gamma\left(a, S^{*}\right)}{\int_{0}^{t} \varphi(a) \Gamma\left(a, S^{*}\right) d W(a)}=K\left(A-S^{*}\right) \Gamma\left(a, S^{*}\right) .
\end{aligned}
$$


If Eq. (14) has a positive root $S^{*}>0$, the corresponding steady state is given by (15). However $\left(R_{0} S^{*}\right)$ is a monotone decreasing function and it decreases from infinity to zero when $S^{*}$ moves from zero to infinity, hence we conclude that (14) has a unique positive root, which provides the steady space size occupied by steady population $q^{*}$.

Proposition 2. The system (13) has a unique positive steady state.

By using the steady state solution, we can rewrite the basic system (13) as a semilinear Cauchy problem. Let us define a new function $u(a, t)$ as $u(a, t):=q(a, t)-q^{*}(a)$ where $q^{*}(a)$ is a stationary solution of (13). Then the system (13) can be written as the following system of $u(a, t)$ :

$$
\begin{aligned}
& \frac{\partial u(a, t)}{\partial a}+\frac{\partial u(a, t)}{\partial t}=-\delta\left(a, q^{*}+G(u)\right)\left(q^{*}(a)+u(a)\right)-q_{a}^{*}(a) \\
& u(0, t)=-K G(u) \\
& u(a, 0)=u_{0}(a):=q_{0}(a)-q^{*}(a) \text { where } \\
& G(u):=\int_{0}^{t} \varphi(a) u(a) d W(a) .
\end{aligned}
$$

Therefore, we can formulate basic model as a semi-linear Cauchy problem in $L^{1}$ as

$$
u^{\prime}(t)=A u(t)+F(u(t))
$$

where the differential operator $A$ and the nonlinear operator $F$ is defined by

$$
\begin{gathered}
(A u)(a):=-\frac{d u(a)}{d a} \\
D(A):=\left\{f \in W^{1,1}(0, t): f(0)=-K G(f)\right\} \\
(F f)(a):=-\delta\left(a, q^{*}+G(f)\right)\left(q^{*}(a)+f(a)-q_{a}^{*}(a)\right)
\end{gathered}
$$

where $D(A)$ denotes the domain of differential operator $A$. The linear operator $A$ generate strongly continuous semigroup $T_{0}(t)=\dot{e}^{t A}$ and compact resolvent (Pazy 1983).

Moreover, under the Assumption, the bounded nonlinear operator $F$ is locally Lipschitz continuous, so a weak local solution of (16) is given by a continuous solution of 


$$
u(t)=T_{0}(t) u_{0}+\int_{0}^{t} T_{0}(t-s) F(u(s)) d W(s)
$$

We define the semi-flow $T(t)$ by $T(t) u_{0}=u(t)$. If $q_{0} \in D(A)$, then the weak solution is given by (19) becomes a classical solution (Pazy 1983). Let $\omega_{0}$ be growth bound of linear semi-group $e^{t A}$ such that $\left|T_{0}(t)\right| \leq e^{\omega_{0} t}$. It follows from (19) that

$$
|u(t)| \leq e^{\omega_{0} t}\left|u_{0}\right|+\int_{0}^{t} e^{\omega_{0}(t-s)}\left\{\bar{\delta}\left(\left|q^{*}\right|+|u(s)|\right)+\left|q_{a}^{*}\right|\right\} d W(s)
$$

Then it is easy to see that

$$
|u(t)| \leq\left(\left|u_{0}\right|+\omega^{-1}\left(\bar{\delta}\left|q^{*}\right|+\left|q_{a}^{*}\right|\right)\right) e^{\left(\omega_{0}+\bar{\delta}\right) t} .
$$

Therefore, the absolute of the weak solution grows at most exponentially, so the local solution can be extended to the global solution defined for $t \in[0, \infty)$. Then the solution of the system (13) can be obtained by $q(a, t)=q^{*}(a)+u(a, t)$, stays in $\Omega$ if $q_{0} \in \Omega$.

\section{References}

Foerster, Von H. 1959. Some remarks on changing population, in "The Kinetics of Cell Cellular Proliferation", F. Stohlman Jr., Ed, Grune and Stratton, New York, 387- 407.

Gard, Thomas C. 1988. Introduction to stochastic differential equation, Marcel Dekker, New York.

Gurtin, M.E. and R. C. MacCamy, 1974. Non-linear age-dependent population dynamics, Arch. Rat. Mech. Anal., 54: 281-300.

Langhaar, H.L.1972. General population theory in the age-time continuum, J. Franklin Inst., 293: $199-214$

Murray, J.D. 2002. Mathematical biology, $3^{\text {rd }}$ Edition, Springer-Verlag, New York, Berlin.

Pazy, A. 1983. Semi groups of linear operators and applications to partial differential equations, Springer-Verlag, New York.

Roughgarden, J., Y. Iwasa and C. Baxter. 1985. Demographic theory for an open marine population with space limited recruitment, Ecology 66: 54-67.

Sharpe, F. R. and A. J. Lotka. 1911. A problem in age distribution, Phil. Magazine, 21: 435 - 438. 